\title{
Screening for genes and subnetworks associated with pancreatic cancer based on the gene expression profile
}

\author{
JIN LONG, ZHE LIU, XINGDA WU, YUANHONG XU and CHUNLIN GE \\ Department of General Surgery, The First Hospital of China Medical University, Shenyang, Liaoning 110001, P.R. China
}

Received April 16, 2015; Accepted February 17, 2016

DOI: $10.3892 / \mathrm{mmr} .2016 .5007$

\begin{abstract}
The present study aimed to screen for potential genes and subnetworks associated with pancreatic cancer (PC) using the gene expression profile. The expression profile GSE 16515 was downloaded from the Gene Expression Omnibus database, which included 36 PC tissue samples and 16 normal samples. Limma package in $\mathrm{R}$ language was used to screen differentially expressed genes (DEGs), which were grouped as up- and downregulated genes. Then, PFSNet was applied to perform subnetwork analysis for all the DEGs. Moreover, Gene Ontology (GO) and REACTOME pathway enrichment analysis of up- and downregulated genes was performed, followed by protein-protein interaction (PPI) network construction using Search Tool for the Retrieval of Interacting Genes Search Tool for the Retrieval of Interacting Genes. In total, 1,989 DEGs including 1,461 up- and 528 downregulated genes were screened out. Subnetworks including pancreatic cancer in PC tissue samples and intercellular adhesion in normal samples were identified, respectively. A total of 8 significant REACTOME pathways for upregulated DEGs, such as hemostasis and cell cycle, mitotic were identified. Moreover, 4 significant REACTOME pathways for downregulated DEGs, including regulation of $\beta$-cell development and transmembrane transport of small molecules were screened out. Additionally, DEGs with high connectivity degrees, such as CCNA2 (cyclin A2) and PBK (PDZ binding kinase), of the module in the protein-protein interaction network were mainly enriched with cell-division cycle. CCNA2 and PBK of the module and their relative pathway cell-division cycle, and two subnetworks (pancreatic cancer and intercellular adhesion subnetworks) may be pivotal for further understanding of the molecular mechanism of PC.
\end{abstract}

Correspondence to: Dr Jin Long, Department of General Surgery, The First Hospital of China Medical University, 155 Nanjing Bei Street, Shenyang, Liaoning 110001, P.R. China

E-mail: jinlongjlq@163.com

Key words: pancreatic cancer, differentially expressed genes, function enrichment, protein-protein interaction network, molecular mechanism

\section{Introduction}

Pancreatic cancer (PC), a digestive system tumor, is one of the most aggressive types of cancer. With a high degree of malignancy, rapid progression and poor prognosis, the 1- and 5-year survival rates of patients with PC are only 8 and $3 \%$, respectively (1). American Cancer Society statistics reported that there were an estimated 36,800 related fatalities and 43,140 new cases of PC in 2010. PC remains the fourth leading cause of cancer-related mortality in the United States, despite advances in detection, chemotherapy and surgery (2). In developing countries, for example, in China, the incidence of $\mathrm{PC}$ has also been markedly increasing during the past several decades, and PC has been ranked the sixth leading cause of death from malignant disease (3).

The inability to detect PC in its early treatable stage may be the critical factor contributing to high mortality. PC is characterized by the lack of notable clinical symptoms and patients often present with symptoms, such as back pain, weight loss, and digestive problems (4). As many as $80 \%$ of newly diagnosed patients with PC are already in the metastatic stage of the disease, which limits the potential for therapeutic intervention (5). At this stage, several epigenetic as well as genetic changes have taken place and result in the silencing of tumor suppressors and overexpression of oncogenes, ultimately leading to tumor progression (6). In recent years, important advances have been made to understand the molecular biology of PC and genetic analyses have verified that the basis of this malignant disease is heterogenous and complex (7). The occurrence and pathogenesis of $\mathrm{PC}$, however, is not yet completely understood.

Similar to the majority of tumors, the development and growth of PC is a multistep process including initiation, progression, invasion and ultimately metastasis. Each step in this process is considered to be driven by the accumulation of genetic alterations (8). Numerous studies involving PC have been conducted in order to identify cancer-causing genes over the past decade, and as a result several cancer-related genes have been identified $(9,10)$. For instance, DPC4, which encodes SMAD family member 4 (SMAD4), is found to be inactivated in $\sim 50 \%$ of all PCs (11). KRAS, an oncogene which is associated with cell survival, proliferation and differentiation, has been identified in $>90 \%$ of patients with PC, with the majority of these being point mutations at codon 12 . In addition, it has been demonstrated that the detection of the KRAS 
mutation may be useful in identifying patients at high risk for developing PC (12). The identification and characterization of cancer-associated genes have increased the understanding of PC development. However, the survival rate has not improved as much in the past years due to the lack of early diagnosis and effective chemotherapeutic treatments. Therefore, identification of genes associated with the development of PC is required.

To test the hypothesis that the FK506-binding protein 51 (FKBP51) may function as a tumor suppressor, Pei et al (13) performed microarray analysis and submitted the expression profile, including 36 pancreatic cancer tissue samples and 16 normal samples, to the Gene Expression Omnibus database (GEO). This previous study was predominantly focused on the functional mechanism of the single gene FKBP51. Based on the gene expression profile submitted by Pei et al (13) and bioinformatics methods, differentially expressed genes (DEGs) between PC tissue samples and normal samples were determined in the present study. Furthermore, functional annotation of DEGs was conducted, followed by the construction of the protein-protein interaction (PPI) network. This study aimed to increase the understanding of the mechanism underlying PC development.

\section{Materials and methods}

Affymetrix microarray data. The expression profiles were accessible at the National Center of Biotechnology Information (NCBI) Gene Expression Omnibus database (http://www.ncbi. nlm.nih.gov/geo) using the series accession number GSE16515, which was deposited by Pei et al (13). This data set was based on the GPL570 platform of [Affymetrix Human Genome U133 Plus 2.0 Array (HG-U133_Plus_2); Affymetrix, Santa Clara, CA, USA] and updated on Aug 22, 2014. A total of 52 chips were divided into 2 groups: $\mathrm{PC}$ tissue samples (T-group, $\mathrm{n}=36$ ) and normal samples (N-group, $\mathrm{n}=16$ ).

Data processing and DEG identification. The probe-level data were firstly transformed into gene expression data. Then background corrections and quartile data normalization were conducted using the robust multiarray average (RMA) in the affy package (Fred Hutchinson Cancer Research Center, Seattle, WA, USA) with default parameters (14).

To screen DEGs between the T-group and N-group, the Limma package (Linear Models for Microarray Data) in $\mathrm{R}$ language was used (15). The raw P-value was adjusted to the false discovery rate based on the Benjamini-Hochberg approach $(16,17)$ using the Limma package (version 3.22.1; Fred Hutchinson Cancer Research Center). DEGs were identified with the cutoff value of FDR $<0.05$ and Ilog (fold change) $\mid>1 \quad(18,19)$.

Subnetwork analysis. Paired Fuzzy SNet (PFSNet) (20) is a powerful method to identify smaller parts of pathways termed subnetworks. Comparison with previously published methods shows that significant subnetworks (and the genes therein) identified by PFSNet are up to 51\% (64\%) more consistent across independent datasets of the same disease phenotypes, even for datasets based on different platforms (20). In order to obtain the genes and subnetworks that may be associated with the biological characteristics of a sample, PFSNet was used in this study to analyze the subnetworks of the genes from the T-group as well as the N-group based on pathways from PathwayAPI (21), which integrated Wikipathways (22), Kyoto Encyclopedia of Genes and Genomes (KEGG) (23) and Ingenuity (24). Steps were conducted as follows: i) The pathways were divided into several subnetworks according to the genes with high expression level; ii) the subnetwork of each group was scored as 1 or 2 , based on the equation as previously described (20); iii) the difference of scores in each group was evaluated by t-test and the subnetworks with significantly different scores were screened out. Parameters in the PFSNet were set as $b=0.5, t_{1}=0.95$, and $t_{2}=0.8$.

Gene ontology (GO) function annotation and pathway enrichment analysis. The Database for Annotation Visualization and Integrated Discovery (DAVID) provides a comprehensive set of functional annotation tools to determined the biological meaning of a large list of genes (25). DAVID was used for GO function annotation and REACTOME pathway enrichment analysis of the up- and downregulated genes, respectively. $\mathrm{P}<0.05$ was selected as the cut-off criterion.

PPI network construction. Search Tool for the Retrieval of Interacting Genes (STRING, http://string-db.org/) is an online database which includes experimental as well as predicted interaction information and comprises $>1,100$ completely sequenced organisms (26). The protein interactions in the STRING database were shown with a confidence score. To identify the interactive associations between the target genes and other genes, the up- and downregulated genes between the T-group and N-group were inputted into STRING and protein pairs with a confidence score $\geq 0.7$ were considered to be significant. Cytoscape (National Institute of General Medical Sciences of the National Institutes of Health, Bethesda, MD, USA) was performed to visualize the PPI network.

The PPI network was complicated; thus, further analysis was required to expose the enriched functional modules of the PPI network using ClusterONE (Clustering with overlapping neighborhood expansion) in Cytoscape (27). Then DAVID was used to annotate the function of genes in each module.

\section{Results}

DEG identification. After data preprocessing, the normalized expression profile data were analyzed using Limma package in $\mathrm{R}$ language. With FDR $<0.05$ and $\mid \log$ (fold change) $\mid>1$, 1,989 DEGs including 1,461 up- and 528 downregulated genes, were screened out in the T-group compared with the N-group.

Subnetwork analysis. In the N-group, 5 significant subnetworks were identified and were shown to be associated with glutathione metabolism (Fig. 1A), leucine and isoleucine metabolism (Fig. 1B), pancreatic cancer (Fig. 1C), calcium signaling pathway (Fig. 1D) and the mitogen-activated protein kinase pathway (Fig. 1E), respectively. Significant subnetworks of genes from the T-group were in association with galactose metabolism (Fig. 2A), alanine, aspartic acid and glutamic acid metabolism (Fig. 2B), intercellular cell adhesion (Fig. 2C) and contraction of vascular smooth muscle (Fig. 2D and E). 

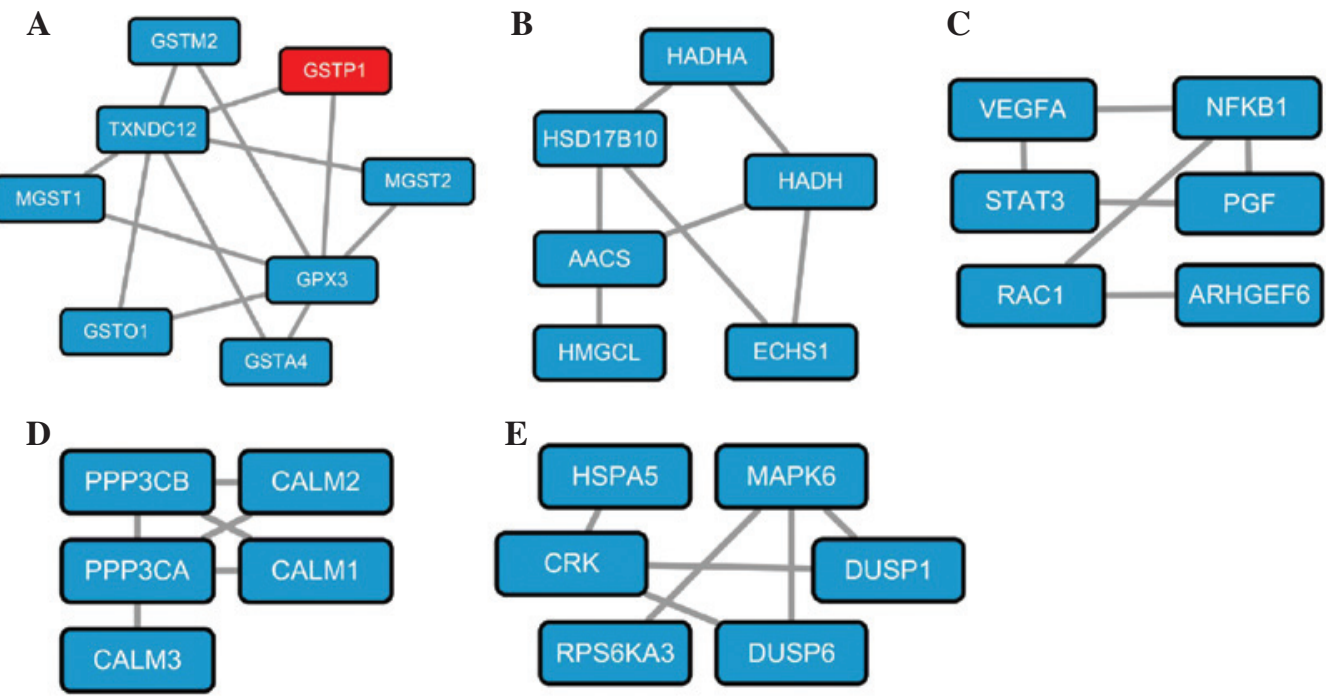

Figure 1. Five significant subnetworks of the N-group, associated with (A) glutathione metabolism (B) leucine and isoleucine metabolism (C) pancreatic cancer (D) calcium signaling pathway and (E) the mitogen-activated protein kinase pathway. Red nodes indicate upregulated genes and blue nodes downregulated genes.

A

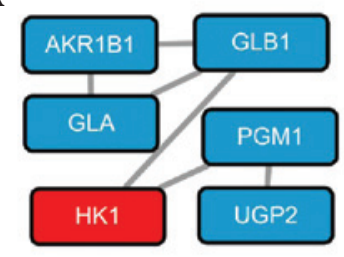

D

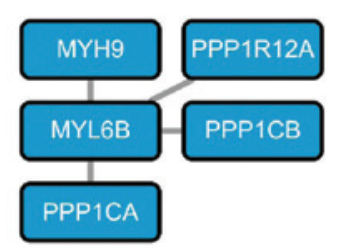

B

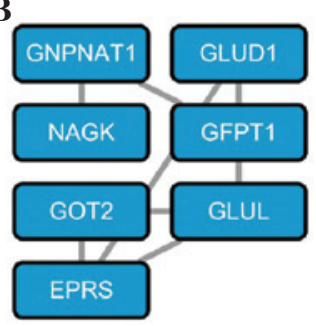

$\mathbf{E}$

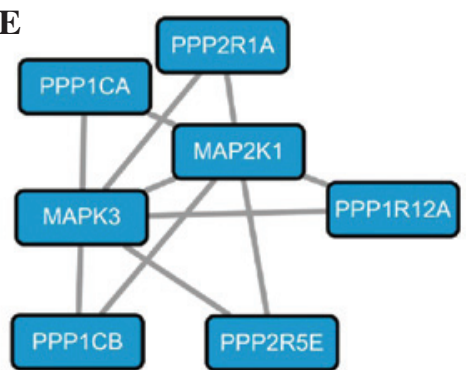

C

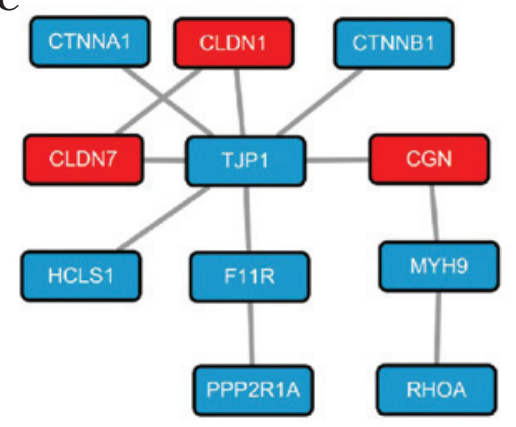

Figure 2. Five significant subnetworks of genes from the T-group, associated with (A) galactose metabolism (B) alanine, (C) aspartic acid and glutamic acid metabolism, (D) intercellular junction and (E) contraction of vascular smooth muscle. Red nodes represent the upregulated genes and the blue nodes represent downregulated genes.

Function and pathway annotation. To gain further insight into the function of the identified DEGs, the online biological classification software DAVID was applied to annotate the DEGs. The upregulated genes were enriched in 14 GO subcategories with the most genes enriched in the cell adhesion pathway (Table I). The downregulated genes were enriched in 9 subcategories with the highest number of genes enriched in the proteolysis pathway (Table I). In addition, 8 significant REACTOME pathways for upregulated genes, such as metabolism of carbohydrates, hemostasis and cell cycle, mitotic were identified (Fig. 3A). Moreover, 4 significant REACTOME pathways for downregulated genes, regulation of $\beta$-cell development, transmembrane transport of small molecules, hemostasis and metabolism of amino acids were screened out (Fig. 3B).
PPI construction and module analysis. The up- and downregulated genes between the T-group and $\mathrm{N}$-group were input into the STRING database to identify the significant interactions with a confidence score of $\geq 0.7$. The PPI network reveals the molecular mechanisms of pancreatic cancer, but it contains too many nodes and interactions to select the useful information. Therefore, the functional modules in the PPI network were mined by ClusterONE. In the current study, the significant module with the lowest P-value for the upregulated DEGs was displayed in Fig. 4A, and 5 DEGs with higher connectivity degrees including cyclin-dependent kinase 1 ( $C D K 1)$, maternal embryonic leucine zipper kinase (MELK), PDZ-binding kinase $(P B K)$, Cyclin A2 (CCNA2) and nucleolar and spindle associated protein 1 (NUSAPI) were included in this module. GO 
Table I. Top gene ontology functional enrichment of up- and downregulated genes.

A, Upregulated

\begin{tabular}{lcc}
\hline Term & Gene count & P-value \\
\hline GO:0007155 cell adhesion & 73 & $<0.001$ \\
GO:0022610 biological adhesion & 73 & $<0.001$ \\
GO:0006955 immune response & 66 & $<0.001$ \\
GO:0006952 defense response & 59 & 0.000001 \\
GO:0042127 regulation of cell proliferation & 58 & 0.001423 \\
GO:0007049 cell cycle & 57 & 0.001696 \\
GO:0009611 response to wounding & 52 & 0.000002 \\
GO:0010033 response to organic substance & 51 & 0.006513 \\
GO:0055114 oxidation reduction & 47 & 0.004401 \\
GO:0022402 cell cycle process & 46 & 0.000678 \\
GO:0008219 cell death & 46 & 0.046477 \\
GO:0016265 death & 46 & 0.047681 \\
GO:0008283 cell proliferation & 43 & 0.000016 \\
GO:0022403 cell cycle phase & 41 & 0.000024 \\
\hline
\end{tabular}

B, Downregulated

\begin{tabular}{lcr}
\hline Term & Gene count & P-value \\
\hline GO:0006508 proteolysis & 33 & 0.000243 \\
GO:0010033 response to organic substance & 24 & 0.001006 \\
GO:0006811 ion transport & 22 & 0.009722 \\
GO:0042592 homeostatic process & 21 & 0.015010 \\
GO:0009611 response to wounding & 20 & 0.000721 \\
GO:0048878 chemical homeostasis & 18 & 0.003062 \\
GO:0009719 response to endogenous stimulus & 17 & 0.000678 \\
GO:0019725 cellular homeostasis & 17 & 0.002910 \\
GO:0009725 response to hormone stimulus & 16 & 0.000718
\end{tabular}

Term, Gene Ontology pathway name; gene counts, number of differentially expressed genes enriched in the Gene Ontology function.

analysis showed that the DEGs in this module were predominantly associated with cell-division cycle (Table II). While, 5 hub genes with the higher degrees including albumin $(A L B)$, carboxypeptidase A1 (pancreatic) $(C P A l)$, colipase, pancreatic $(C L P S)$, epidermal growth factor $(E G F)$ and complement component 5 (C5) were identified in the significant module with the lowest P-value for the downregulated DEGs with the lowest P-values, which is shown in Fig. 4B. Moreover, the DEGs in this module predominantly participated in biological processes, such as response to wounding, endogenous stimulus and regulation of cell proliferation (Table II).

\section{Discussion}

$\mathrm{PC}$ is one of the leading causes of cancer-related mortality worldwide; however, the molecular mechanisms of PC progression remain unclear. With the rapid expansion of knowledge on genomics, emerging evidence suggests that the initiation, progression, invasion and metastasis of PC are generally caused by the differential expression of genes. In the present study, a total of 1,989 DEGs including 1,461 up- and 528 downregulated genes were screened out. In line with the results of the study by Pei et al (13), FKBP5 was identified as one of the downregulated genes in the PC samples. To understand the interaction of these DEGs, a PPI network was constructed and the significant module with the lowest P-value for upregulated genes with the top 5 nodes of $C D K 1$, $M E L K, P B K, C C N A 2$ and NUSAP1 and the module with the lowest P-value for downregulated genes with the top 5 nodes of ALB, CPA1, CLPS, EGF and C5 were identified. Among all these proteins, $C D K 1, A L B, C P A 1, C L P S$ and $E G F$ were verified to be associated with PC (28-31). Moreover, the association of $M E L K$ and $C 5$ with $\mathrm{PC}$ have been demonstrated in certain studies (32-34). However, according to the present results, $C C N A 2$ and $P B K$, which have not previously been directly associated with $\mathrm{PC}$, may be pivotal for the initiation and progression of PC. In addition, certain subnetworks may be important in PC via the differential expression of genes involved, such as the subnetwork directly associated with PC and the subnetwork associated with intercellular cell adhesion. 


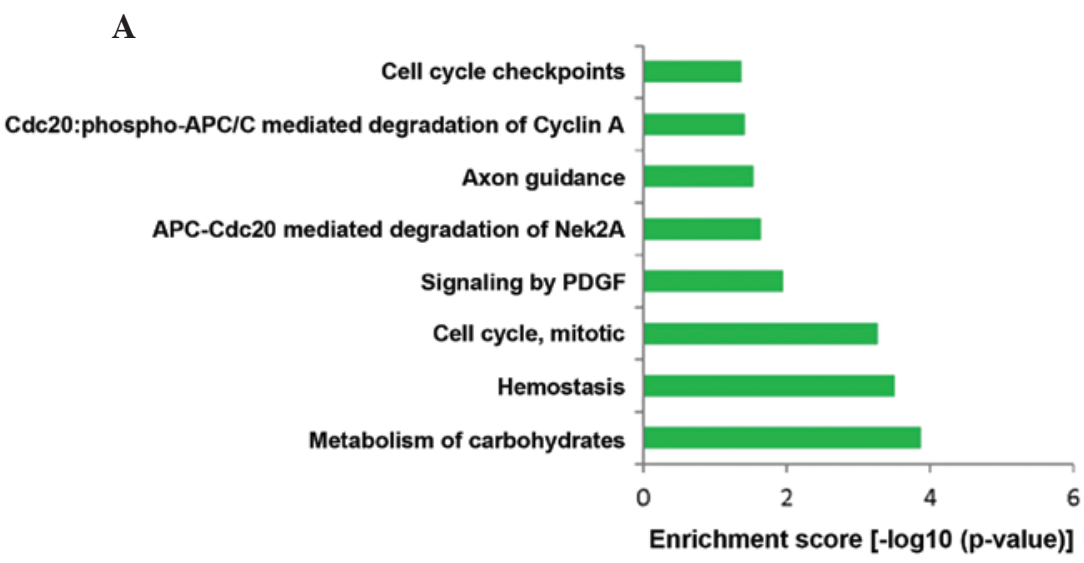

B

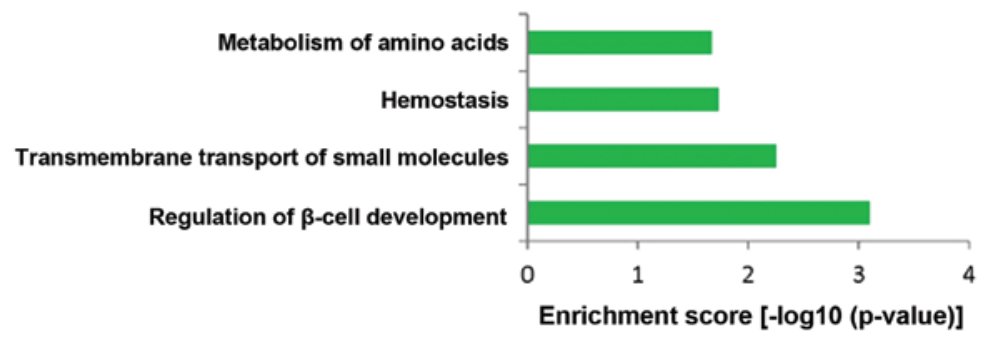

Figure 3. Pathway enrichment for differentially expressed genes. (A) Upregulated genes and (B) downregulated genes.
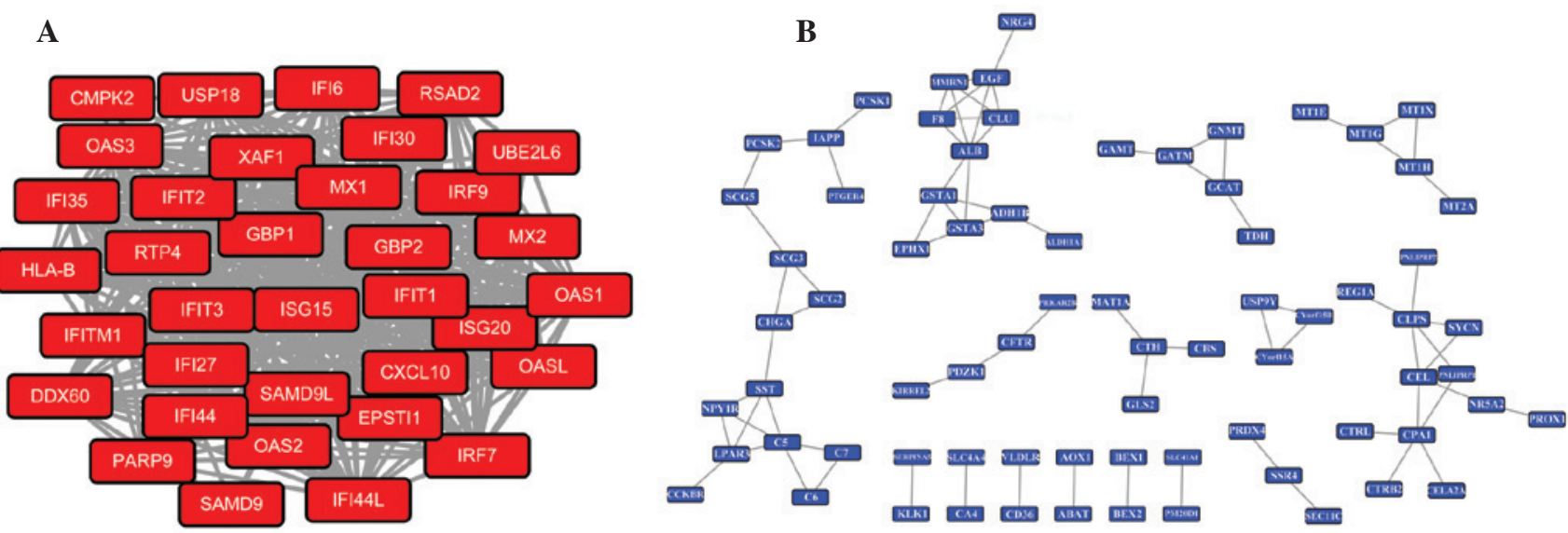

Figure 4. Significant module of (A) upregulated genes and (B) downregulated genes. Red nodes represent the upregulated DEGs. While blue nodes represent downregulated genes.

Cyclins are a family of proteins that control the progression of cells through the cell cycle by activating cyclin-dependent kinases (CDK) (35). As a member of the cyclin family, CCNA2 is produced at the onset of DNA synthesis in proliferating somatic cells and is critical in cell cycle progression by regulation of transition from G1 to S phase (36). Genetic variants of CCNA2, which may affect the function of the encoded protein by changing gene expression or by altering the protein structure, are found to significantly increase the risk of cancer development in a tissue-specific manner, such as colon, liver and lung cancer (37). In addition, Gao et al (38) reported that CCNA2 was a biomarker for the prognosis of breast cancer and a promising target for developing novel strategies to prevent or even reverse tamoxifen resistance. In addition, the expression of CCNA2 may aid in monitoring tamoxifen efficacy and directing personalized therapies in patients with breast cancer (38). Few previous studies have focused on the association of CCNA2 and PC, while high throughput bioinformatics analysis in the present study indicates that CCNA2 may be important for the initiation and development of PC. In the present study, CCNA2 was found to be upregulated in $\mathrm{PC}$ tissue samples, and functional analysis demonstrates that CCNA2 was predominantly enriched in the cell cycle pathway and participates in biological processes, such as regulation of cell proliferation, regulation of cell cycle, cell cycle checkpoint and mitosis. These findings were concordant with those of previous studies $(39,40)$. Therefore, it was hypothesized that $C C N A 2$ may be important in the pathogenesis of PC via regulation of the cell cycle and mitosis, which may further influence tumor occurrence. 
Table II. Top Gene Ontology annotation of up- and downregulated genes in the significant module with the lowest P-value of the protein-protein interaction network.

A, Upregulated

\begin{tabular}{lcr}
\hline Term & Gene count & P-value \\
\hline GO:0007049 cell cycle & 44 & $<0.001$ \\
GO:0022402 cell cycle process & 38 & $<0.001$ \\
GO:0022403 cell cycle phase & 37 & $<0.001$ \\
GO:0000279 M phase & 36 & $<0.001$ \\
GO:0000278 mitotic cell cycle & 32 & $<0.001$ \\
GO:0051301 cell division & 29 & $<0.001$ \\
\hline
\end{tabular}

B, Downregulated

\begin{tabular}{lcr}
\hline Term & Gene count & P-value \\
\hline GO:0006508 proteolysis & 15 & 0.000234 \\
GO:0009611 response to wounding & 11 & 0.000134 \\
GO:0010033 response to organic substance & 11 & 0.001507 \\
GO:0009725 response to hormone stimulus & 9 & 0.000256 \\
GO:0009719 response to endogenous stimulus & 9 & 0.000495 \\
GO:0042127 regulation of cell proliferation & 9 & 0.026531 \\
\hline
\end{tabular}

PBK, also known as PDZ-binding kinase, is a mitotic protein kinase and its encoding gene, $P B K$ was found to be upregulated in PC tissue samples. Studying upregulated kinases in cancer may provide important clues as to the mechanism of malignant conversion $(41,42)$. PBK is phosphorylated in vitro by $\mathrm{Cdc} 2$-cyclin $\mathrm{B}$ at a site in the amino terminus (Thr 9) which is implicated in the binding of $\alpha$-tubulin, and then localizes to mitotic spindles and spindle poles during metaphase (43). Studies regarding $P B K$ have demonstrated that the expression of $P B K$ is regulated by cell cycle-specific transcription factors, such as E2F and CREB/ATF, and knockdown expression of $P B K$ may lead to cytokinetic dysfunction in breast cancer $(44,45)$. Ayllón et al (46) suggested that $P B K$ is involved in DNA damage sensing and repair via phosphorylating c-H2AX. Nandi et al (47) confirmed that with western immunoblotting and immunoprecipation, and yeast two-hybrid analysis, PBK can directly interact with p53, downregulate its expression and attenuate $\mathrm{G} 2 / \mathrm{M}$ checkpoint in fibrosarcoma cells, which was hypothesized to be a plausible explanation for the role of $P B K$ in augmenting tumor cell growth. Similarly, the GO-biological process enrichment in the present study predicted that $P B K$ was predominantly associated with nuclear division, cell division, $\mathrm{M}$ phase of mitotic cell cycle, and $P B K$ with higher connectivity degree in the module with the lowest $\mathrm{P}$-value of upregulated genes was enriched in cell division cycle. Based on these results, it was inferred that $P B K$ may influence the occurrence of $\mathrm{PC}$ by regulating the mitotic cell cycle and other biological processes.

Intercellularcell adhesion determines the polarity of cells and participates in the maintenance of tissues (48). Several studies have shown that cell-cell adhesiveness is generally reduced in human cancer, which may result in influences as follows: Reduced intercellular adhesiveness allows loss and disruption of cell-cell adhesion, resulting in destruction of histological structure, which is the morphological hallmark of malignant tumors (48). Conversely, reduced intercellular adhesiveness is also indispensable for cancer invasion and metastasis (49). In line with the previous studies, subnetworks associated with intercellular cell adhesion were found to be significant in the T-group, and the majority of the genes in this subnetwork were identified to be upregulated in PC. Accordingly, intercellular cell adhesion may be important in the progression of PC.

The significant subnetwork directly associated with PC consisted of six genes, VEGFA, NFKB1, STAT3, PGF, RAC1 and $A R H G E F 6$. Expression levels of the majority of these genes were identified to be significantly higher in PC samples and this subnetwork is directly involved in PC via the differential expression of genes involved. For instance, STAT3 is confirmed to be vital in anti-pancreatic cancer effects through its contributions to the positive feedback loop between reactive oxygen species and autophagy (50). The concentration of $P G F$ is found to be significantly increased in pancreatic carcinoma compared with tumor-free tissue (51). Moreover, activation of RAC1-dependent superoxide generation leads to PC cell proliferation and inhibition of $R A C 1$ may be a potential therapeutic strategy (52). Hence, as demonstrated, subnetworks directly associated with pancreatic cancer may be crucial in the pathogenesis of PC.

In conclusion, the results of this study may increase the understanding of the mechanism of the occurrence and development of PC. CCNA2 and PBK of the module and their relative pathway cell-division cycle may be pivotal for understanding the molecular mechanism of PC. In addition, two subnetworks (pancreatic cancer subnetwork and intercellular adhesion subnetwork) may be highly associated with PC. However, the whole study was conducted based on bioinfor- 
matics methods, and the conclusions have not been verified by corresponding experiments yet. Thus, further experiments are urgently required to confirm the results of this study.

\section{References}

1. Moore MJ, Goldstein D, Hamm J, Figer A, Hecht JR, Gallinger S, Au HJ, Murawa P, Walde D, Wolff RA, et al: Erlotinib plus gemcitabine compared with gemcitabine alone in patients with advanced pancreatic cancer: A phase III trial of the national cancer institute of canada clinical trials group. J Clin Oncol 25 : 1960-1966, 2007.

2. Jemal A, Siegel R, Xu J and Ward E: Cancer statistics, 2010. CA Cancer J Clin 60: 277-300, 2010.

3. Long J, Luo GP, Xiao ZW, Liu ZQ, Guo M, Liu L, Liu C, Xu J, Gao YT, Zheng Y, et al: Cancer statistics: Current diagnosis and treatment of pancreatic cancer in Shanghai, China. Cancer Lett 346: 273-277, 2014.

4. Chan A, Diamandis EP and Blasutig IM: Strategies for discovering novel pancreatic cancer biomarkers. J Proteomics 81 : 126-134, 2013.

5. Pliarchopoulou K and Pectasides D: Pancreatic cancer: Current and future treatment strategies. Cancer Treat Rev 35: 431-436, 2009.

6. Sato $\mathrm{N}$ and Goggins $\mathrm{M}$ : The role of epigenetic alterations in pancreatic cancer. J Hepatobiliary Pancreat Surg 13: 286-295, 2006.

7. Jones S, Zhang X, Parsons DW, Lin JC, Leary RJ, Angenendt P, Mankoo P, Carter H, Kamiyama H, Jimeno A, et al: Core signaling pathways in human pancreatic cancers revealed by global genomic analyses. Science 321: 1801-1806, 2008

8. Logsdon CD, Simeone DM, Binkley C, Arumugam T, Greenson JK, Giordano TJ, Misek DE, Kuick R and Hanash S: Molecular profiling of pancreatic adenocarcinoma and chronic pancreatitis identifies multiple genes differentially regulated in pancreatic cancer. Cancer Res 63: 2649-2657, 2003.

9. Wang B, Sun S and Liu Z: Analysis of dysregulation of immune system in pancreatic cancer based on gene expression profile. Mol Biol Rep 41: 4361-4367, 2014.

10. Kern SE: Molecular genetic alterations in ductal pancreatic adenocarcinomas. Med Clin North Am 84: 691-695, 2000.

11. Shin SH, Kim SC, Hong SM, Kim YH, Song KB, Park KM and Lee YJ: Genetic alterations of K-ras, p53, c-erbB-2 and DPC4 in pancreatic ductal adenocarcinoma and their correlation with patient survival. Pancreas 42: 216-222, 2013.

12. Fryzek JP, Garabrant DH, Schenk M, Kinnard M, Greenson JK and Sarkar FH: The association between selected risk factors for pancreatic cancer and the expression of $\mathrm{p} 53$ and $\mathrm{K}$-ras codon 12 mutations. Int J Gastrointest Cancer 37: 139-145, 2006.

13. Pei H, Li L, Fridley BL, Jenkins GD, Kalari KR, Lingle W, Petersen G, Lou Z and Wang L: FKBP51 affects cancer cell response to chemotherapy by negatively regulating Akt. Cancer Cell 16: 259-266, 2009.

14. Dorsey ER, Constantinescu R, Thompson JP, Biglan KM, Holloway RG, Kieburtz K, Marshall FJ, Ravina BM, Schifitto G, Siderowf A and Tanner CM: Projected number of people with Parkinson disease in the most populous nations, 2005 through 2030. Neurology 68: 384-386, 2007.

15. Delhomme N, Padioleau I, Furlong EE and Steinmetz LM: easyRNASeq: A bioconductor package for processing RNA-Seq data. Bioinformatics 28: 2532-2533, 2012.

16. Singh B, Ronghe AM, Chatterjee A, Bhat NK and Bhat HK: MicroRNA-93 regulates NRF2 expression and is associated with breast carcinogenesis. Carcinogenesis 34: 1165-1172, 2013.

17. Chand Y and Alam MA: Network biology approach for identifying key regulatory genes by expression based study of breast cancer. Bioinformation 8: 1132-1138, 2012.

18. Benjamini Y and Hochberg Y: Controlling the false discovery rate: A practical and powerful approach to multiple testing. J R Stat Soc Series B Stat Methodol: 289-300, 1995.

19. Benjamini Y: Discovering the false discovery rate. J R Stat Soc Series B Stat Methodol 72: 405-416, 2010.

20. Lim K and Wong L: Finding consistent disease subnetworks using PFSNet. Bioinformatics 30: 189-196, 2014.

21. Soh D, Dong D, Guo Y and Wong L: Consistency, comprehensiveness, and compatibility of pathway databases. BMC Bioinformatics 11: 449, 2010
22. Kelder T, van Iersel MP, Hanspers K, Kutmon M, Conklin BR, Evelo CT and Pico AR: WikiPathways: Building research communities on biological pathways. Nucleic Acids Res 40 (Database Issue): D1301-D1307, 2012.

23. Kanehisa M, Goto S, Sato Y, Furumichi M and Tanabe M: KEGG for integration and interpretation of large-scale molecular data sets. Nucleic Acids Res 40 (Database Issue): D109-D114, 2012.

24. Krämer A, Green J, Pollard J Jr and Tugendreich S: Causal analysis approaches in ingenuity pathway analysis. Bioinformatics 30 : 523-530, 2014.

25. Hu Y, Hu Y, Liu D, Yu J and Liu D: Screening and bioinformatics analysis of differentially expressed genes in hyperplastic scar. Nan Fang Yi Ke Da Xue Xue Bao 34: 939-944, 2014 (In Chinese).

26. Franceschini A, Szklarczyk D, Frankild S, Kuhn M, Simonovic M, Roth A, Lin J, Minguez P, Bork P, von Mering C and Jensen LJ: STRING v9.1: Protein-protein interaction networks, with increased coverage and integration. Nucleic Acids Res 41 (Database Issue): D808-D815, 2013.

27. Shannon P, Markiel A, Ozier O, Baliga NS, Wang JT, Ramage D, Amin N, Schwikowski B and Ideker T: Cytoscape: A software environment for integrated models of biomolecular interaction networks. Genome Res 13: 2498-2504, 2003.

28. Feldmann G, Mishra A, Bisht S, Karikari C, Garrido-Laguna I, Rasheed Z, Ottenhof NA, Dadon T, Alvarez H, Fendrich V, et al: Cyclin-dependent kinase inhibitor Dinaciclib (SCH727965) inhibits pancreatic cancer growth and progression in murine xenograft models. Cancer Biol Ther 12: 598-609, 2011.

29. Hempen PM, Kurpad H, Calhoun ES, Abraham S and Kern SE: A double missense variation of the BUB1 gene and a defective mitotic spindle checkpoint in the pancreatic cancer cell line Hs766T. Hum Mutat 21: 445, 2003.

30. Zhang P, Zou M, Wen X, Gu F, Li J, Liu G, Dong J, Deng X, Gao J, Li X, et al: Development of serum parameters panels for the early detection of pancreatic cancer. Int J Cancer 134: 2646-2655, 2014.

31. Renouf D and Moore M: Evolution of systemic therapy for advanced pancreatic cancer. Expert Rev Anticancer Ther 10: 529-540, 2010.

32. Kusakai G, Suzuki A, Ogura T, Kaminishi M and Esumi H: Strong association of ARK5 with tumor invasion and metastasis. J Exp Clin Cancer Res 23: 263-268, 2004.

33. Kokkinakis DM, Liu X and Neuner RD: Modulation of cell cycle and gene expression in pancreatic tumor cell lines by methionine deprivation (methionine stress): Implications to the therapy of pancreatic adenocarcinoma. Mol Cancer Ther 4: 1338-1348, 2005

34. Michl P, Buchholz M, Rolke M, Kunsch S, Löhr M, McClane B, Tsukita S,Leder G, Adler G and Gress TM: Claudin-4: A new target for pancreatic cancer treatment using Clostridium perfringens enterotoxin. Gastroenterology 121: 678-684, 2001.

35. Dun B, Sharma A, Xu H, Liu H, Bai S, Zeng L and She JX Transcriptomic changes induced by mycophenolic acid in gastric cancer cells. Am J Transl Res 6: 28-42, 2013.

36. Gong D, Pomerening JR, Myers JW, Gustavsson C, Jones JT, Hahn AT, Meyer T and Ferrell JE Jr: Cyclin A2 regulates nuclear-envelope breakdown and the nuclear accumulation of cyclin B1. Curr Biol 17: 85-91, 2007.

37. Kim DH, Park SE, Kim M, Ji YI, Kang MY, Jung EH, Ko E, Kim Y, Kim S, Shim YM and Park J: A functional single nucleotide polymorphism at the promoter region of cyclin A2 is associated with increased risk of colon, liver and lung cancers. Cancer 117: 4080-4091, 2011

38. Gao T, Han Y, Yu L, Ao S, Li Z and Ji J: CCNA2 is a prognostic biomarker for ER+ breast cancer and tamoxifen resistance. PLoS One 9: e91771, 2014

39. Kokkinakis DM, Liu XY and Neuner RD: Modulation of cell cycle and gene expression in pancreatic tumor cell lines by methionine deprivation (methionine stress): Implications to the therapy of pancreatic adenocarcinoma. Mol Cancer Ther 4: 1338-1348, 2005.

40. Roderick HL and Cook SJ: $\mathrm{Ca}^{2+}$ signalling checkpoints in cancer: Remodelling $\mathrm{Ca}^{2+}$ for cancer cell proliferation and survival. Nat Rev Cancer 8: 361-375, 2008.

41. Baselga J and Arribas J: Treating cancer's kinase 'addiction'. Nat Med 10: 786-787, 2004

42. Bettencourt-Dias M, Giet R, Sinka R, Mazumdar A, Lock WG, Balloux F, Zafiropoulos PJ, Yamaguchi S, Winter S, Carthew RW, et al: Genome-wide survey of protein kinases required for cell cycle progression. Nature 432: 980-987, 2004.

43. Gaudet S, Branton D and Lue RA: Characterization of PDZ-binding kinase, a mitotic kinase. Proc Natl Acad Sci USA 97: 5167-5172, 2000 . 
44. Park JH, Lin ML, Nishidate T, Nakamura $\mathrm{Y}$ and Katagiri T: PDZ-binding kinase/T-LAK cell-originated protein kinase, a putative cancer/testis antigen with an oncogenic activity in breast cancer. Cancer Res 66: 9186-9195, 2006.

45. Nandi AK and Rapoport AP: Expression of PDZ-binding kinase $(\mathrm{PBK})$ is regulated by cell cycle-specific transcription factors E2F and CREB/ATF. Leuk Res 30: 437-447, 2006.

46. Ayllón V and O'connor R: PBK/TOPK promotes tumour cell proliferation through p38 MAPK activity and regulation of the DNA damage response. Oncogene 26: 3451-3461, 2007.

47. Nandi AK, Ford T, Fleksher D, Neuman B and Rapoport AP: Attenuation of DNA damage checkpoint by PBK, a novel mitotic kinase, involves protein-protein interaction with tumor suppressor p53. Biochem Biophys Res Commun 358: 181-188, 2007.

48. Hirohashi S and Kanai Y: Cell adhesion system and human cancer morphogenesis. Cancer Sci 94: 575-581, 2003.
49. Saiki I: Cell adhesion molecules and cancer metastasis. Jpn J Pharmacol 75: 215-242, 1997.

50. Gong J, Muñoz AR, Chan D, Ghosh R and Kumar AP: STAT3 down regulates LC3 to inhibit autophagy and pancreatic cancer cell growth. Oncotarget 5: 2529-2541, 2014.

51. Heukamp I, Kilian M, Gregor JI, Kiewert C, Schimke I, Kristiansen G, Walz MK, Jacobi CA and Wenger FA: Impact of polyunsaturated fatty acids on hepato-pancreatic prostaglandin and leukotriene concentration in ductal pancreatic cancer-is there a correlation to tumour growth and liver metastasis? Prostaglandins Leukot Essent Fatty Acids 74: 223-233, 2006.

52. Aravindan S, Delma CR, Thirugnanasambandan SS, Herman TS and Aravindan N: Anti-pancreatic cancer deliverables from sea: First-hand evidence on the efficacy, molecular targets and mode of action for multifarious polyphenols from five different brown-algae. PLoS One 8: e61977, 2013. 